\title{
MEDIOS DE PRUEBA EN EL PROCESO CIVIL ESPAÑOL JUSTIFICADOS EN PRESUNCIONES: LA PRUEBA INDICIARIA, CIRCUNSTANCIAL O INDIRECTA
}

\author{
EVIDENCE IN THE SPANISH CIVIL PROCESS JUSTIFIED IN ASSUMPTIONS: THE \\ INDICIARY, CIRCUMSTANTIAL OR INDIRECT EVIDENCE
}

Antonio María Lorca Navarrete* Universidad del País Vasco

\section{Resumen}

La Ley de Enjuiciamiento Civil N. ${ }^{\circ} 1 / 2000$ introduce al entendimiento de la presunción (artículos 385 y siguientes de dicho dispositivo normativo), donde su omnipresencia abre insospechadas posibilidades a la práctica judicial justificada en su tránsito inequívoco a través de la prueba indiciaria, indirecta o circunstancial.

Palabras clave: Presunciones; indicio; prueba; Código Procesal Civil.

\section{Abstract}

The Ley de Enjuiciamiento Civil N. ${ }^{\circ} 1 / 2000$ introduce the understanding of the presumption (articles 385 and following of the act), in which its omnipresence opens up unsuspected possibilities to the justified judicial practice in its unequivocal transit through the indiciary evidence, indirect or circumstantial

Keywords: Presumption; circumstantial evidence; evidence; Spanish Civil procedure Act.

Doctor en Derecho por la Universidad de Granada, España. Profesor en la Universidad del País Vasco, España. Director del Instituto Vasco de Derecho Procesal y de la Revista Vasca de Derecho Procesal y Arbitraje. 
En la Ley de Enjuiciamiento Civil española no se alude expresamente a la prueba indiciaria, indirecta o circunstancial. El desinterés e indiferencia hacia la misma ya fue exhibido en el siglo XIX cuando se indicó que «en las aulas y en nuestros tratadistas de derecho se hacen, a lo sumo, ligeras indicaciones sobre tan difícil materia. De suerte, que para adquirir algunas noticias sobre ella es preciso recurrir a autores extranjeros» (López, 1879, p. 6).

En la actualidad, y tras la entrada en vigor de la Ley de Enjuiciamiento Civil del 2000, es posible aludir, aun cuando sea con el carácter de inédito para un texto procesal civil, a la presunción que permite sustentar o justificar la prueba indiciaria, indirecta o circunstancial.

Las anteriores afirmaciones se agigantan aún más si se tiene en cuenta que, a la conceptuación de la vigente Ley de Enjuiciamiento Civil como Código General del Proceso (artículo 4 de la Ley de Enjuiciamiento Civil), se une el devenir histórico de la procesalística en España debido a la nula atención que se ha prestado a la prueba indiciaria, indirecta o circunstancial en el ámbito del proceso civil.

Se ha dicho, a modo de pauta para comprender en su exacto sentido lo que se acaba de indicar, que «con toda la magnitud y respeto a la obra del profesor Serra Domínguez Normas de presunción en el Código civil (...), su reducción semántica y sistemática del indicio a la voz «afirmación base» y el poco juego que le otorga al mismo a lo largo de su obra no ayuda a una perspectiva metafórica y pedagógicamente celular de esta entidad lógica» (Muñoz, 2000, p. 332).

Con la entrada en vigor de la Ley de Enjuiciamiento Civil N. ${ }^{\circ}$ 1/2000, en cambio, al tiempo que se accede al entendimiento y comprensión de la presunción (artículos 385 y siguientes de la Ley de Enjuiciamiento Civil), su omnipresencia abre insospechadas posibilidades a la práctica judicial justificada en su tránsito inequívoco a través de la prueba indiciaria, indirecta o circunstancial.

Para comprender, por tanto, en sus exactos términos la prueba indiciaria, indirecta o circunstancial en el ámbito del proceso civil, la Ley de Enjuiciamiento Civil nos obliga a transitar a través de las presunciones. De ahí que resulte obligado un examen particularizado la cuestión relativa al nexo existente entre presunción y prueba indiciaria, indirecta o circunstancial. $\mathrm{O}$, lo que es lo mismo, acudir en primer término, al indicio. 


\section{EL INDICIO O PRUEBA INDICIARIA (O, TAMBIÉN, INDI- RECTA O CIRCUNSTANCIAL)}

La presunción implica considerar como ciertos los indicios -o hechos bases de la presunción-. Al respecto, se ha indicado lo siguiente:

[...] que entendemos por indicio todo hecho conocido que demuestra la existencia de otro desconocido. El indicio viene a ser como un signo. Entre el indicio pues, el hecho que indica, ha de mediar la relación de necesidad entre el signo y los significados. De otra suerte cabe error, siendo imposible afirmar nada con absoluta certeza.» (López, 1879, p. 75).

Mas en particular, el indicio «es un hecho que está en relación tan íntima con otro hecho, que un juez llega del uno al otro por medio de una conclusión natural» (Muñoz, 2000, p. 335) aunque «el concepto de «relación íntima» no deja de ser un concepto indeterminado» (Muñoz, 2011, p. 3) de modo que la relación del indicio «con otros indicios, forma una arborescencia que al socaire de esta descripción metafórica desemboca en una presunción, que en el lenguaje taxonómico sería como el ancestro común de todos los indicios» (Muñoz, 2011, p. 3).

La interrogante que se plantea es la siguiente: «广Y por qué no dar nombre a todos estos indicios?» (Muñoz, 2011, p. 3). Sobre el particular, se ha indicado que

[...] una taxonomía indiciaria es básicamente expositiva en cuanto se limita a dar cuenta de los indicios sin atribuir conjuntos o síndromes para cada thema probandi en concreto (cuestión esta que representa un paso más adelante que corresponde de lleno a la semiótica probática) y exponencial, en cuanto que los indicios que presenta pueden hallarse ubicados en diferentes thema probandi, tanto parecidos como disimiles. (Muńoz, 2011, p. 3).

En definitiva, [e]stamos en un presente, tratamos de 〈lograr〉 el pasado con unos hechos (de circum stare: que están alrededor) que no son los buscados. Pero estos, con el empleo de la racionalidad y las reglas de la experiencia, nos indican el buscado (el hecho buscado no está representado ni observado por el juez; lo que tenernos es un hecho que lo señala. Es decir, el que nos interesa no está en el expediente, lo que hay hecho que lo indica, lo señala).» (Parra, 2007, 685).

\section{EL INDICIO Y SU RELACIÓN CON LA PRESUNCIÓN}

El indicio en su relación con la presunción se proyecta en los siguientes ámbitos. El primero supone que para poder «presumir» a partir de uno 
o varios indicios, hay que concretar las exigencias de «carácter formal y material» en que ha de concurrir el indicio que permita precisamente "presumir» un determinado hecho o hechos con relevancia probatoria para el proceso civil. En semejante contexto

[...] la filosofía enseña, y el sentido común patentiza, que donde quiera se presente un efecto, ha debido antes existir una causa suficiente y, al contrario, que toda causa produce sus efectos.

De aquí que pueda deducirse sin temor de engaño la existencia de la causa por la del efecto, y viceversa. Pero un mismo efecto puede provenir de muy distintas causas, y al revés, una causa produce muy diferentes efectos (López, 1879, p. 103).

Tras lo indicado, se podría afirmar que desde la perspectiva apuntada relativa a las exigencias de "carácter formal» del indicio se requiere que se exprese cuáles son los indicios que se estiman plenamente acreditados para "presumir» un determinado hecho o hechos con relevancia probatoria para el proceso civil. Por su parte, desde la perspectiva de las exigencias de «carácter material» del indicio se requiere que estén plenamente acreditados y que sean plurales o siendo único que posea una singular potencia acreditativa. También se requiere que los indicios estén interrelacionados y que cuando sean varios se refuercen entre sí. En tal sentido, «en la prueba indiciaria concurren varios indicios, o sea varios hechos indicadores y un solo indicado. Este suele recibir el nombre de principal y aquellos de accesorios o circunstanciales. Por ello, se llama también esta prueba circunstancial» (López, 1879, p. 79).

Por su parte, el segundo de los ámbitos aludidos atańe a que el indicio o indicios le permita al juez "presumir» una concreta «deducción o inferencia» en la que quede explicitado el razonamiento realizado a partir del indicio respecto del que es imprescindible el control de su racionalidad,es decir, que no solamente no sea arbitraria, absurda e infundada, sino que responda plenamente a las reglas de la lógica y de la experiencia.

En tercer término, el indicio le permite «presumir» al juez cuando de los indicios fluya, según una concreta "deducción o inferencia», el dato a acreditar siempre que exista entre el indicio y el dato a acreditar un enlace preciso y directo según las reglas del criterio humano.

El examen correlativo de tales exigencias supone que la presunción implica que el tribunal ha de llevar a cabo una valoración crítica y una argumentación elaborada en base al indicio ya conocido que, en modo 
alguno, es una técnica de conocimiento «al primer contacto». De ahí «la importancia que la doctrina otorga a la prueba indiciaria en el desarrollo del proceso, cualquiera que éste sea, reconociendo su actitud para formar la convicción del juzgador» (Cordón, 2012, p. 44).

\section{EL MEDIO DE PRUEBA}

El medio de prueba es el indicio que actúa indirectamente o circunstancialmente con el fin de acreditar un determinado hecho. Que luego, y a partir del indicio el juez presuma su acreditación (la acreditación del indicio o de lo obtenido de modo indirecto o circunstancial), es sino la consecuencia consistente en que «el indicio no es susceptible de llevar al ánimo sino presunciones» (López, 1879, p. 155).

Pero, la presunción no es un medio de prueba. A decir verdad, más que un medio de prueba, la presunción es el resultado de un proceso lógico al que la Ley de Enjuiciamiento Civil le reconoce, en ciertas condiciones, la idoneidad para lograr la prueba de un concreto hecho [«à dire il vero, più che un mezzo di prova rappressentano il risultato di procedimento lógico, al quale la legge riconosce, a certe condizioni, l’idioneità a raggiungere la prova di un fatto ignoto» (Arieta, De Santis, Montesano]. No es de extrañar que se haya propugnado la "consideración de las presunciones como método de prueba» (Alvárez, 2007, p. 45).

En la práctica, esa nueva afirmación - la presumida acerca de la certeza de otro hecho indicio - sirve para distinguir las verdaderas presunciones, que suponen la formación de nuevas afirmaciones presumidas, de las presunciones aparentes, que incluyen en la nueva afirmación otras ya comprendidas en la afirmación base.

En efecto, la presunción se sustenta en «un componente eminentemente fáctico (sería el indicio) [que] (...), ante la falta de prueba directa al respecto, resulta preciso acudir al mecanismo de la presunción, como cauce probatorio, toda vez que en virtud de aquel, se permite que el juzgador, a partir de un hecho probado o admitido en el proceso (sería el indicio), pueda presumir la certeza de otro hecho, si entre el admitido y el presunto existe un enlace preciso y directo según las reglas del criterio humano» (Loyola, 2014, pp. 307-308). O sea, a decir del magistrado ponente Hoya, que la presunción es un 
mecanismo en virtud del cual y por la existencia de un vínculo de causalidad de un hecho denominado base (sería el indicio), demostrado por otro medio de prueba, se deduce el denominado hecho consecuencia, que tiene la misma eficacia probatoria que la obtenida directamente, distinguiéndose dentro de las presunciones las denominadas legales o de derecho praesumptiones iuris seu legis, cuando la deducción la formula la ley, y las judiciales o de hombre praesumptiones hominis seu iudicis». (Lorca, 2011, p. 13).

\section{CLASES DE PRESUNCIONES}

Conviene, entonces, destacar que en la vigente Ley de Enjuiciamiento Civil el indicio permite «presumir» dos tipos de presunciones. De un lado, las presunciones legales y, de otro, las presunciones judiciales.

En relación con las presunciones legales o de derecho hay que tener en cuenta que «existiendo una de tales presunciones, el que la tenga a su favor sólo ha de afirmar y probar el indicio del que la ley deduce el hecho que se presume» (Schönke, 1950, pp. 204 y 205). Son presunciones legales porque es la propia ley las que las establece como sucede cuando la ley presume la legitimidad de los hijos cuando ha concurrido el indicio de su nacimiento durante el matrimonio o dentro de un determinado tiempo posterior a la ruptura matrimonial. O la archiconocida presunción constitucional de inocencia según la cual, salvo indicios de culpabilidad, se presume siempre la inocencia, básica para comprender el proceso penal en la actualidad.

La característica de las presunciones legales estriba no tanto en que «dispensan de la prueba del hecho presunto" — tal y como indica el artículo 385.1. de la Ley de Enjuiciamiento Civil - cuanto que la certeza del hecho indicio o circunstancial, del que parte la presunción, ha quedado establecido legalmente mediante su admisión. Se evita, de ese modo, que se formulen presunciones partiendo de un hecho indicio inconcreto o indeterminado lo que explica que «las presunciones establecidas por la ley admitirán la prueba en contrario, salvo en los casos en que aquélla — la ley- expresamente lo prohíba» (artículo 385.3. de la Ley de Enjuiciamiento Civil). Por tanto, la presunción legal admite «prueba en contrario». Y la «prueba en contrario» puede

dirigirse tanto a probar la inexistencia del hecho presunto como a demostrar que no existe en el caso de que se trate, el enlace que ha de haber entre el hecho que se presume y el hecho probado o admitido que fundamenta la presunción» (artículo 385.2. de la Ley de Enjuiciamiento Civil). 
De no mediar "prueba en contrario», la viabilidad de la prueba indiciaria, indirecta o circunstancial se halla asegurada.

Por su parte, las presunciones judiciales o de hecho son las que establece el propio tribunal. En ellas y a partir de un hecho indicio admitido, el tribunal puede - es facultativo- presumir la certeza, a los efectos del proceso civil, de otro hecho o circunstancia, si entre el hecho indicio admitido o demostrado y el presunto existe un enlace preciso y directo según las reglas del criterio humano. Las denominadas «reglas del criterio humano» son las reglas de la lógica que permiten al tribunal determinar cuál es el enlace más adecuado ["preciso y directo»] al caso concreto. En concreto, «el Juez, en virtud de las máximas de experiencia, estima que se da el grado de probabilidad necesario para suponer ocurrido un hecho, y con ello como realizada su prueba (prueba prima facie)» (Schönke, 1950 p. 225).

Todo lo cual se traduce en la adopción de dos medidas. La primera, atañe a que la sentencia debe «incluir el razonamiento en virtud del cual el tribunal ha establecido la presunción» que no ha de ser ilógico, irracional ni arbitrario (artículo 386.1. de la Ley de Enjuiciamiento Civil). La segunda, en servirse de modelos de las ciencias empíricas y de los cánones de la lógica y de la metodología de las ciencias que permitan oponer la prueba en contrario por cuanto la parte perjudicada pueda probar la inexistencia del hecho presunto (artículo 386.2. de la Ley de Enjuiciamiento Civil). Siendo lo relevante de las presunciones judiciales el aspecto relativo a la inferencia o enlace lógico entre los hechos indicio y consecuencia, constituyendo la pieza clave la razonabilidad de la deducción siempre que la inferencia no adolezca de ilegalidad, error, falta de lógica o arbitrariedad.

Esas presunciones judiciales eran las que se regulaban en el Código Civil. No en vano el «artículo 386 de la Ley de Enjuiciamiento Civil — sustitutorio del artículo 1.253 del código civil. actualmente derogado- [se halla] referido a las presunciones judiciales» (Picazo, 2011, p. 99).

En consecuencia, de no mediar la «prueba en contrario» de la inexistencia del hecho presunto, la viabilidad, nuevamente, de la prueba indiciaria, indirecta o circunstancial civil se halla asegurada. 


\section{BIBLIOGRAFÍA}

Arieta, G., De Santis, F. \& Montesano, L. (2018). Corso base di diritto processuale civile. Setima edizione. Roma-Salerno: Wolters Kluwers. Cedam, pp. 141, 324, 371, 372, 409.

Álvarez, P. (2007). La prueba por presunciones. Particular referencia a su aplicación judicial en supuestos de responsabilidad extracontractual. Granada p. 45.

Cordón, J. C. (2012). Prueba indiciaria y presunción de inocencia en el proceso penal. Publicación del Instituto Vasco de Derecho Procesal. San Sebastián, p. 44.

Lorca Navarrete (2011). Jurisprudencia procesal civil comentada de las Audiencias Provinciales vascas. Estudio procesal civil de los autos y sentencias de las Audiencias Provinciales vascas a partir de la entrada en vigor de la Ley de Enjuiciamiento Civil N. ${ }^{\circ} 1 / 2000$. Año 2001. Volumen I. Edición Instituto Vasco de Derecho Procesal. San Sebastián 2011, pp. 65, 176, 364.

López, S. (1879). La prueba de indicios. (Prólogo). Madrid, pp. 75, 78, 79, 103 y 155.

López, S. (1901). Principios fundamentales del procedimiento civil y criminal. Tomo II. Madrid, pp. 22, 23, 51.

Loyola, A. M. (2014). En A. Ma. Lorca Navarrete. Jurisprudencia procesal civil comentada de las Audiencias Provinciales Vascas. Estudio procesal civil de los autos y sentencias de las Audiencias Provinciales vascas a partir de la entrada en vigor de la Ley de Enjuiciamiento Civil N. ${ }^{\circ}$ 1/2000. Año 2004. Volumen IV. Edición Instituto Vasco de Derecho Procesal. San Sebastián, pp. 307- 308

Muñoz, L. (2000) Fundamentos de prueba judicial civil. LEC N. ${ }^{\circ} 1 / 2000$. Editorial J. $\mathrm{M}^{\mathrm{a}}$. Bosch. Barcelona.

Muñoz L. (Martes 8 de febrero de 2011). Taxonomía indiciaria. La Ley. Año XXXII. Número 7564.

Muñoz L. (2016) La prueba de indicios en el proceso judicial. Análisis para juristas, detectives, periodistas, peritos y policías. La Ley. Wolters Kluwers. Madrid.

Parra, J. (2007). Manual de derecho probatorio. $16^{\circ}$ edición. Bogotá: Librería Ediciones del profesional.

Picazo F. J. (2011). A. Ma . Lorca Navarrete. Jurisprudencia procesal civil comentada de las Audiencias Provinciales vascas. Estudio procesal civil de los autos y sentencias de las Audiencias Provinciales vascas a partir de la entrada en vigor de la Ley de Enjuiciamiento Civil $N .^{\circ} N .^{\circ} 1 / 2000$. Año 2001. Volumen I. Edición Instituto Vasco de Derecho Procesal. San Sebastián, p. 99.

Schönke, A. (1950). Derecho procesal civil. Barcelona: Bosch, Casa Editorial, pp. 204,205 y 225. 